\title{
El Rol de la Miel en los Procesos Morfofisiológicos de Reparación de Heridas
}

\author{
Rol of the Miel in the Morphophysiologic Process of the Reparation Wounds
}

\author{
Carolina Schencke ${ }^{*, * *}$; Bélgica Vásquez ${ }^{* * * *}$; Cristian Sandoval ${ }^{*, * * * *, * * * * * *}$ \& Mariano del Sol ${ }^{*, * * * * *, * * * * * * * * *}$
}

SCHENCKE, C.; VÁSQUEZ, B.; SANDOVAL, C. \& DEL SOL, M. El rol de la miel en los procesos morfofisiológicos de reparación de heridas. Int. J. Morphol., 34(1):385-395, 2016.

RESUMEN: Los beneficios de la miel de abeja (Apis mellifera) se conocen desde hace miles de años y ha sido utilizada en la antigüedad por su valor nutritivo y medicinal siendo "redescubierta" por la medicina moderna debido a sus importantes propiedades antibacterianas en heridas infectadas. Nuevos estudios han argumentado a favor de la capacidad de la miel para interactuar con la compleja maquinaria celular y llevar a cabo la reparación de los tejidos. Por este motivo, se realizó una revisión de los contenidos actuales de las propiedades de la miel y su rol en los procesos morfofisiológicos de reparación de heridas. De esta manera se abarcó la acción antibacteriana, anti-oxidante y anti-inflamatoria de sus componentes. Así mismo, se revisaron las propiedades físicas y de desbridamiento, la importancia del origen floral y ubicación geográfica en el valor medicinal de los diferentes tipos de miel. Los estudios sugieren que los efectos de la miel son el resultado de una actividad combinada, donde sus componentes interactúan de manera sinérgica para lograr su función terapéutica. Su acción la realiza en todas las etapas del proceso morfofisiológico de reparación logrando acelerar la cicatrización de heridas. El origen florar y ubicación geográfica de la miel son factores importantes a considerar, ya que ésta varía en su composición y, por lo tanto, también su valor medicinal.

\section{PALABRAS CLAVE: Heridas; Quemaduras; Cicatrización; Miel.}

\section{INTRODUCCIÓN}

La miel es definida como una sustancia dulce, no fermentada, producida por las abejas (Apis mellifera) que recogen y procesan el néctar de las flores o de las secreciones de ciertas especies de plantas. Las abejas, transforman y combinan esta sustancia con otras específicas propias que finalmente almacenan y maduran en panales.

Los beneficios de la miel se conocen desde hace miles de años y ha sido utilizada en la antigüedad por su valor nutritivo y medicinal. Las primeras evidencias del consumo de la miel aparecen en pinturas rupestres del Mesolítico, unos 6.000 años A.C. y su uso como medicamento unos 2.500 años A.C. por los sumerios en Mesopotamia. Entre los usos médicos, desde la antigüedad la miel ha servido en el cuidado de heridas. Los antiguos egipcios, los asirios, los chinos, los griegos y los romanos utilizaban la miel para tratar heridas. En los papiros de Eberts y Smith, que datan de 1500 A.C. ya se aconsejaba tratar con miel las heridas. Hipócrates, en su obra "Consideraciones sobre el tratamiento de las heridas", recomienda curarlas con miel (Al-Waili et al., 2011; Tan et al., 2012; Eteraf-Oskouei \& Najafi, 2013; Zbuchea, 2014; Jull et al., 2015).

Después de haber cumplido un papel importante en la tradición médica de muchos pueblos, la miel fue "redescubierta" por la medicina moderna debido a sus importantes propiedades bactericidas en heridas infectadas con bacterias multiresistentes a los antibióticos (Godebo et al., 2013; Melake et al., 2015). Desde entonces, numerosos estudios han centrado su interés en demostrar que las propiedades biológicas y físicas de la miel le confieren una gran eficacia en el tratamiento de heridas de diversas etiologías (Al-Waili et al.; Jull et al.) y que su uso ofrece un tratamiento natural alternativo en la población mundial que reducen el costo de

* Doctorado en Ciencias Morfológicas, Facultad de Medicina, Universidad de La Frontera, Temuco, Chile.

** CONICYT-PCHA/Doctorado Nacional/2014-21141130.

**** Universidad de Tarapacá, Arica, Chile.

***** Centro de Investigación CIMA, Facultad de Odontología, Universidad de La Frontera, Temuco, Chile.

****** CONICYT-PCHA/Doctorado Nacional/2015-21150991

******* Centro de Excelencia en Ciencias Morfológicas y Quirúrgicas (CEMyQ), Universidad de La Frontera, Temuco, Chile.

******** Centro de Investigación en Ciencias Biomédicas, Universidad Autónoma de Chile, Chile. 
los tratamientos modernos (Gupta et al., 2011; Schencke et al., 2013; Mujalde et al., 2014).

La miel presenta un conjunto de propiedades que contribuyen significativamente en el proceso de cicatrización de heridas. Muestra un efecto anti-bacteriano en heridas susceptibles a infecciones o infectadas, actividad anti-oxidante que reduce la alta concentración de radicales libres y especies reactivas de oxígeno (ROS) producidas en la etapa inflamatoria, actividad anti-inflamatoria, anti-edematosa y exudativa que reduce rápidamente el dolor, edema y exudado de las heridas, desbridamiento del tejido necrótico estimulando la formación de tejido de granulación sano, estimula la contracción de la herida favoreciendo el cierre de la misma y muestra propiedades cicatrizantes que reducen al mínimo su apariencia (Suguna et al., 1993; Velnar et al., 2009; Molan, 2011; Yaghoobi et al., 2013; Oryan et al., 2016).

En acuerdo con lo reportado, este estudio tuvo como objetivo realizar una revisión general sobre los contenidos actuales de las propiedades curativas y cicatrizantes de la miel en heridas, con énfasis en lesiones por quemadura. Además, se discutió el rol de la miel en los procesos fisiológicos de reparación de heridas y se revisó la importancia del origen floral y ubicación geográfica, en el valor medicinal de diferentes tipos de miel.

\section{Propiedades anti-bacterianas de la miel}

En quemaduras, sus propiedades anti-bacterianas hacen que sea un agente natural adecuado para facilitar el control microbiano de heridas infectadas. Para ello, cuenta con un conjunto de factores que afectan directamente a los microorganismos patógenos, como bacterias gram positivas y negativas, aerobias y anaerobias, incluyendo a los tipos de bacterias multiresistentes a los antibióticos (Estrada et al., 2005; Lay-flurrie, 2008; Nishio et al., 2016). Estos factores comprenden la acción del peróxido de hidrógeno, la alta osmolaridad, la acidez y factores no basados en peróxido, tales como metilglioxal (MGO) (Adams et al., 2008), el péptido antimicrobiano de abeja defensina-1 (Kwakman et al., 2010), hidroximetifurfural (HMF) (Nafea et al., 2011) y compuestos fenólicos, tales como flavonoides (Cushnie \& Lamb, 2005). Las acciones antimicrobianas indirectas incluyen aumento en la producción de linfocitos, anticuerpos, citoquinas y el fortalecimiento del propio sistema inmunológico (Molan, 2001; Subrahmanyam et al., 2007; Khoo et al., 2010; Al-Waili et al.).

Actualmente, pocos antibióticos son eficaces frente a bacterias multiresistentes. Así, varios estudios han combinado la miel con fármacos antimicrobianos comerciales
(Jenkins \& Cooper, 2012; Müller et al., 2013). Éstas combinaciones son ventajosas y comúnmente utilizadas en tratamientos, principalmente porque proporcionan un amplio espectro de actividad debido, fundamentalmente, al efecto multidiana o por retrasar/suprimir la aparición de una población resistente a fármacos (Eliopoulos \& Eliopoulos, 1988; Ulrich-Merzenich et al., 2010).

Asímismo, otros estudios han reportado sustancias que tienen la capacidad de potenciar respuestas inmunes específicas y no específicas, tales como, concanavalina A (Talebi et al., 1995) y fitohemaglutinina (Oudrhiri et al., 1988), lipopolisacáridos (LPS), algunos virus (Butchko et al., 1978) y ciertas drogas (Archambault et al., 1989). que combinados con miel pueden potenciar su capacidad bactericida. En este sentido, Abuharfeil et al. (2010) demostraron que la miel de abeja, suplementada con LPS, tiene un efecto significativo en la capacidad reductora de fagocitos no estimulados.

\section{Propiedades anti-oxidante y anti-inflamatoria de la miel}

Se cree que el efecto negativo en los seres humanos de diversos factores ambientales estaría mediada por la generación de ROS. Parte de la protección contra ROS se proporciona por los antioxidantes endógenos o por una serie de diferentes compuestos contenidos en la dieta. Así, la miel, como fuente de antioxidantes, es eficaz contra el deterioro oxidativo (Blasa et al., 2006).

La actividad anti-oxidante intrínseca de la miel, incluyen la inactivación y la supresión de ROS por los fagocitos en los tejidos inflamados y disminución del estrés oxidativo por la injuria térmica mediante el control de los radicales libres que se forman en la quemadura de la herida. La supresión de ROS, inhibe a los fibroblastos y conduce a una reducción de la fibrosis y cicatrización hipertrófica. Según Tonks et al. (2001), tanto la miel de Manuka como mieles productoras de peróxido de hidrógeno demostraron ser inhibidores de la producción de ROS por células monocíticas (MM6).

Numerosos estudios han demostrado que las mieles más oscuras tendrían mayor poder anti-oxidante por ser más ricas en compuestos fenólicos como flavonoides y taninos. Es conocido que los compuestos fenólicos contribuyen significativamente a la capacidad anti-oxidante de la miel, pero no son los únicos responsables de esta propiedad. Otros compuestos asociados a la actividad antioxidante de la miel son el ácido ascórbico, vitaminas del complejo B, tocoferoles, catalasa, superóxido dismutasa, glutatión reductasa, péptidos, aminoácidos y selenio (Meda et al., 2005; Singh et al., 2012; Jubri et al., 2013; Pourreza, 2013; 
Saric et al., 2013; Vandamme et al., 2013). Gheldof et al. (2002) nombran ácidos orgánicos, tales como glucónico, cítrico y málico. Estos ácidos son metales quelatos y, por tanto, pueden mejorar sinérgicamente la acción de otros antioxidantes, tales como, los fenoles de la miel. También, se ha encontrado la presencia de bajas concentraciones de ácido salicílico, conocido por su capacidad de neutralizar radicales libres (Venema et al., 1996). De la misma manera, diferentes cantidades y tipos de minerales pueden influir en la actividad anti-oxidante de las mieles. El contenido mineral puede variar de aproximadamente $0,04 \%$ en mieles claras a $0,2 \%$ en algunas muestras de mieles oscuras (RodriguezOtero et al., 1994).

Según Gheldof et al. (2002), la capacidad antioxidante de la suma de las fracciones es inferior a la capacidad anti-oxidante de toda la miel. Por lo tanto, la capacidad anti-oxidante de la miel es probablemente el resultado de las interacciones sinérgicas entre los distintos componentes.

Junto con lo anterior, las evidencias sostienen que la miel tiene un efecto favorable sobre la regeneración de heridas, contribuyendo de manera significativa en el proceso de cicatrización no solo a través de su propiedad anti-oxidante, sino que también, a través de su capacidad anti-inflamatoria y de regulación de esta etapa, lo cual se puede evidenciar en la evaluación de la evolución local de la herida (Zbuchea).

La cicatrización de heridas es un proceso de regeneración de tejidos que incluye la inflamación como un primer paso. A veces, estos procesos de curación son inhibidos por terapias con drogas que lo retrasan, en consecuencia, se podrían utilizar nuevos agentes anti-inflamatorios que no afecten estos procesos. Por otro lado, aunque la inflamación es parte del proceso normal de la cicatrización, puede hacer inconfortable la herida y de difícil manejo y, si se prolonga en el tiempo, puede evitar que el tejido de reparación de la herida continúe los procesos de curación (Hadagali \& Chua, 2014). En acuerdo con lo anterior, un producto natural como la miel es una buena alternativa para este fin, ya que no produce los efectos secundarios de algunas drogas y propicia la cicatrización (Molan \& Rhodes, 2015). Un buen número de ensayos clínicos apoyan esta propiedad de la miel, informando la reducción de los síntomas de la inflamación después de la aplicación tópica de la miel en las heridas, con disminución del edema y exudado (Subrahmanyam et al., 2001; Yaghoobi et al.; Zbuchea; Calderón et al., 2015).

Cabe destacar, que la miel tiene una actividad antiinflamatoria directa, ya que la disminución de la inflamación no solo se debe a un efecto secundario de la actividad anti-bacteriana de la miel en la eliminación de bacterias que causan la inflamación. Esta actividad anti-inflamatoria está bien documentada, incluye varios mecanismos, entre los cuales se encuentran la inhibición del complemento, la infiltración de leucocitos, la producción de citoquinas inflamatorias e inhibición de la producción de óxido nítrico y fagocitosis por los macrófagos (Velnar et al.; Molan \& Rhodes). Los componentes fenólicos de la miel también pueden estar involucrados en la actividad anti-inflamatoria, ya que se ha demostrado que puede inhibir la producción de la citoquina inflamatoria factor de necrosis tumoral alfa (TNF- $\alpha$ ). Además, se ha descrito, en la miel de Manuka, la presencia de una proteína llamada Apalbúmina 1 (Apa-1), un potente inhibidor de la fagocitosis en macrófagos. Su mecanismo de acción se realiza mediante el bloqueo del receptor de manosa en los fagocitos, que es el detonador para la fagocitosis (Bean et al., 2012).

\section{Propiedades físicas y de desbridamiento de la miel}

Además de sus propias acciones anti-bacterianas, anti-oxidantes y anti-inflamatorias, la miel crea una barrera física y un medio ambiente local húmedo, debido a su alta viscosidad por concentraciones elevadas de azúcar y al drenaje constante de los fluidos por osmosis desde el lecho de la herida (Molan, 2009). Esto, favorece la cicatrización de las heridas por quemaduras debido a que ellas se curan más rápido en un ambiente húmedo en lugar de un ambiente seco y con formación de costra (Winter, 1962; Kandil et al., 1987; Molan, 2011). Un ambiente húmedo asegura el crecimiento de células epiteliales, promueve el acercamiento de los fibroblastos a los márgenes de la herida, así como la falta de adherencia de los apósitos a la herida, dando lugar a cambios fácil y sin dolor, sin el riesgo de romper epitelio recién formado (Molan, 2011). Además, las características de este entorno local permiten el desbridamiento autolítico eliminando el tejido necrótico y la escara. La miel tiene una acción estimulante sobre el plasminógeno en el lecho de la herida, aumentando la actividad de la enzima plasmina que digiere la fibrina que se une a restos en la superficie de la herida, pero no digiere la matriz extracelular de colágeno, que es necesaria para la reparación de los tejidos (Molan, 2001).

\section{Propiedades cicatrizantes de la miel}

Nuevas pruebas han argumentado a favor de la capacidad de la miel para interactuar con la compleja maquinaria celular y llevar a cabo la reparación de los tejidos. Diferentes estudios han demostrado que la miel es capaz de promover la angiogénesis, granulación y epitelización, estimular linfocitos y fagocitos, inducir la expresión de marcadores moleculares de reparación de tejidos y la activación de queratinocitos (Burlando \& Cornara, 2013). 
Junto con lo anterior, la producción de IL-6 y TNF- $\alpha$ por macrófagos y otros tipos celulares en la herida son esenciales para el proceso de cicatrización y resolución de la infección. Estudios in vitro han obtenido pruebas acerca de los mecanismos de acción de varios tipos de miel de abejas en células de piel, logrando estimular células monocíticas humanas al activar la producción de citoquinas. Un estudio realizado por Majtán et al. (2010), sobre los queratinocitos y fragmentos de piel demostró que la miel de Acacia indujo la expresión de citocinas (TNF$\alpha$, IL1- $\beta$ y TGF- $\beta$ ) logrando efectos inmunomoduladores y activadores de la metaloproteinasa de matriz 9 (MMP9). Esta última enzima es capaz de degradar el colágeno tipo IV y está implicada en la desorganización de la membrana basal durante el proceso de reepitelización. De esta manera la miel activa queratinocitos y modula la MMP-9 logrando acelerar el proceso de cicatrización de heridas.

Una de las teorías por las cuales la fase inflamatoria logra avanzar a otra fase de cicatrización se explica por la acción del peróxido de hidrógeno, el cual es proporcionado en forma continua por la enzima peroxidasa presente en la miel. De esta manera, logra ser anti-bacteriano y fisiológicamente no tóxico, ya que puede ser perjudicial cuando se añade en altas concentraciones. Los niveles producidos por la miel diluida son aproximadamente 1000 veces menor que en soluciones de enjuague. A esta concentración el peróxido de hidrógeno puede actuar a nivel intracelular como "mensajero", capaz de promover respuestas y estimular la expresión de genes tempranos de crecimiento importantes en la cicatrización de heridas. Es probable que de esta manera logre contribuir a la modulación de células no inflamatorias adyacentes, tales como los fibroblastos. Éste podría ser uno de los principales mecanismos de la miel para estimular la cicatrización de heridas; sin embargo, se necesita más investigación para confirmarla (Lusby et al., 2002).

Para evaluar parámetros de curación dérmica, se ha estudiado el efecto de la miel en la resistencia a la tracción, el contenido de hidroxiprolina para reflejar la síntesis de colágeno, el contenido de ADN para reflejar la fibroplasia, el contenido de ácido urónico y hexosamina que constituyen los glicosaminoglicanos necesarios para la cicatrización de heridas, entre otros.

Bajo este contexto, Suguna et al. (1992) demostraron que se produce un aumento significativo en el contenido total de colágeno en los tejidos de granulación de las heridas cuando son tratados con miel vía tópica o sistémica. Estos autores señalaron que los resultados podrían deberse a que los aminoácidos arginina y el ácido glutámico presentes en la miel podrían suministrar el precursor, prolina, para la síntesis de colágeno y su maduración, acelerando la cicatrización de heridas y mejorando la resistencia a la tracción. Posteriormente, Suguna et al. (1993) observaron en este mismo modelo, que el tratamiento de heridas con miel produce un marcado incremento en el contenido de ADN de los tejidos de granulación reflejando la hiperplasia de las células y un incremento concomitante en proteínas y colágeno debido a que los fibroblastos sintetizan activamente sustancia fundamental, el substrato sobre el que se establece el colágeno. De esta manera, estos resultados se correlacionan positivamente con lo señalado por Aljady et al. (2000), al demostrar que la aplicación tópica y oral de miel en heridas estimula la función de los fibroblastos, con un nivel máximo del contenido de ADN para el día 8 post herida; estimula el depósito de colágeno en el tejido granular, mostrando niveles de hidroxiprolina significativamente mayores al grupo control entre los días 12 y 16 post herida y, mejorar la síntesis de glicosaminoglicanos al demostrar que los niveles más altos de ácido urónico y hexosamina fueron observados en los animales tratados con miel tópica y oral. Estos son los primeros componentes de la matriz extracelular para ser sintetizados durante la cicatrización de heridas y formar la plantilla para el colágeno y la deposición de elastina.

\section{Rol de la miel en los procesos fisiológicos de repara- ción de heridas}

Los procesos fisiológicos de reparación de heridas pueden ser divididos en tres etapas o fases superpuestas: inflamatoria, proliferativa y de remodelación. En la etapa inflamatoria, la miel estimula los monocitos para liberar citoquinas inflamatorias incluyendo TNF- $\alpha$ IL-6, IL-1ß y óxido nítrico que pueden inducir la síntesis de colágeno por los fibroblastos (Raynaud et al., 2013) e iniciar y amplificar los procesos inflamatorios (Hussein et al., 2012). Varios estudios sugieren que existen componentes dentro de algunos tipos de miel que pueden estimular células monocíticas. Esto se puede explicar por el tipo de proteínas presentes, la cuales son altamente glicosiladas (Brownlee, 1995; Tonks et al., 2001). Estudios realizados por Majtán et al. (2006), demostraron que la glicoproteína Apa-1 es un componente regular de la miel y del polen de abeja y estimula a macrófagos para liberar el TNF. La fracción proteica de la glicoproteína sería la responsable de la estimulación de este factor por macrófagos peritoneales murinos. Además, encontraron que la miel nativa fue capaz de estimular la secreción de TNF- $\alpha$ de los macrófagos murinos, mientras que la miel desproteinizada no tuvo ningún efecto. Por otro lado, Tonks et al. (2007) aislaron una proteína de $58 \mathrm{kDa}$ de la miel de Manuka, el cual es un componente responsable de la inducción de citokinas monocíticas humanas, distinta a la ya aislada Apa1. Estos 
resultados sugieren que el efecto inmunoestimulador de la miel se basa en su contenido proteico, específicamente, de glicoproteinas. Sin embargo, en su conjunto, la miel tiene un papel modulador en la fase inflamatoria de la cicatrización de la herida para que inicie una inflamación activa, pero controlada, evitando que la inflamación se desarrolle en un estado crónico o exagerado. Junto con lo anterior, la miel actúa eliminando bacterias y residuos de la herida debido a sus propiedades anti-bacteriana, antiinflamatoria, anti-oxidante y de debridación durante la fase inflamatoria (Hussein et al.; Raynaud et al.)

La etapa proliferativa o de granulación, presenta angiogénesis y fibroplasia dérmica, junto con el inicio de la reepitelización y la contracción de la herida. La estimulación de la angiogénesis por la miel suministra el oxígeno necesario en el proceso de curación de las herida (Molan, 2002; Rossiter et al., 2010). La alta presión osmótica y el peróxido de hidrógeno contenido en niveles bajos en la miel estimula el desarrollo de nuevos capilares (Molan, 2002; Nakajima et al., 2013), el crecimiento y la proliferación de los fibroblastos y miofibroblastos y promueve la síntesis de colágeno y la reepitelización (Rossiter et al.; Boekema et al., 2013; Al-Jadi et al., 2014).

En la etapa de remodelación, el colágeno es remodelado y realineado a lo largo de líneas de tensión y las células que ya no son necesarias se eliminan por apoptosis. Subrahmanyam et al., han informado que la miel puede reducir las cicatrices y contracturas en los pacientes con quemaduras y además mejora la remodelación de las heridas cutáneas. Tan et al., observaron que el uso de miel en el tratamiento de heridas reduce la costra y produce formaciones cicatriciales delgadas.

\section{Importancia del origen floral y geográfico en las pro- piedades de la miel}

Hay que tener en consideración que debido a que las abejas tienen diferente comportamiento nutricional y recogen las sustancias nutritivas de diversas plantas y lugares, las mieles producidas tendrán distintas composiciones. Por lo tanto, diversos tipos de miel tienen diferente valor medicinal dando lugar a distintos efectos sobre la cicatrización de heridas (Oryan et al.). Para la determinación del origen floral es necesario analizar el polen o realizar estudios melisopalinológico, (Anklam, 1998).

Como fue mencionado anteriormente, hay numerosas investigaciones que señalan que la miel tiene una actividad antibacteriana de amplio espectro, sin embargo, existen variaciones en la potencia debido a las diferencias en su origen floral, ubicación geográfica, condi- ciones climáticas, entre otros factores (Al-Waili et al.; Lu et al., 2014). Algunos ejemplos de lo señalado, son las investigaciones realizadas por Molan \& Rhodes, quienes demostraron que la miel de Manuka de Nueva Zelanda, que proviene del arbusto Leptospermum scoparium, tiene una gran actividad anti-bacteriana que no solo se debe al peróxido de hidrógeno presente, sino que además, al componente llamado metilglioxal, un potente inhibidor del crecimiento bacteriano ( $\mathrm{Lu}$ et al., 2013). Por su parte, la miel de Tualang de Malasia presenta mejores resultados que su control (hidrofibra e hidrofibra de plata) con respecto a la reducción del crecimiento bacteriano de Pseudomonas aeruginosa en herida de quemadura de espesor completo en ratas (Khoo et al.). Por otra parte, Ahmed \& Othman (2013) compararon algunas propiedades y características bioquímicas con la miel de Manuka y observaron que sus diferencias incluyen compuestos fenólicos, flavonoides y HMF más altos, lo que le confiere mayor eficacia que la miel de Manuka contra algunas cepas de bacterias gram negativas en las heridas por quemaduras.

Por su parte, la miel monofloral de Ulmo (Eucryphia cordifolia) proveniente de Chile, también ha demostrado tener gran poder bactericida y eficacia en el tratamiento de heridas. Sherlock et al. (2010), sostienen que esta miel tiene un efecto antimicrobiano más potente frente a Staphylococcus aureus, Escherichia coli y Pseudomona aeroginosa, cuando es comparada con miel de Manuka. En modelos animales, el tratamiento con esta miel en heridas por quemaduras mostró ser eficaz en el control bacteriano y en la disminución de la inflamación y edema, favoreciendo una rápida cicatrización al ser comparada con el control. Así mismo, estudios clínicos en pacientes con úlceras venosas han reportado gran efectividad en el tratamiento del uso tópico de miel de Ulmo asociado a la administración oral de ácido ascórbico (Calderón et al.).

Dadas sus particulares propiedades, en Chile se ha creado una patente para el uso de un extracto de miel monofloral de Ulmo, rico en compuestos fenólicos, capaz de inhibir el crecimiento in vitro de bacterias patógenas humanas como Staphylococcus aureus, Pseudomona aeruginosa, Escherichia coli, entre otras, y poseer actividad fungicida y fungistática sobre hongos de ambientes de los géneros Mucor, Rhizopus, Aspergillus, Candida y Penicillium (Montenegro \& Ortega, 2011). Los usos están orientados a la desinfección de superficies mediante un producto de limpieza de uso doméstico, así como también, a la aplicación tópica o sistémica como bactericida y fungicida en seres vivos, tales como animales y el hombre, con un fin terapéutico. 
El origen botánico y geográfico de la miel es un factor que también influye en su actividad anti-oxidante (Frankel et al., 1998). La miel de Manuka de New Zealand presenta un gran contenido de compuestos fenólicos existiendo una fuerte correlación entre la actividad anti-oxidante y el contenido de fenoles totales (Alzahrani et al., 2012). Resultados similares fueron observados con cuatro tipos de mieles monoflorales recogidas en la región oriental de Rumania (Dobre et al., 2010) y otros lugares como la miel de Acacia recolectada en Croacia (Krpan et al., 2009). Por su parte, Pontis et al. (2014) encontraron una correlación positiva entre el color y contenido de flavonoides con la capacidad antioxidante de diez muestras de miel multifloral del estado de Roraima, Brasil. Chua et al. (2013), estudiaron la propiedad anti-oxidante comparativa de tres mieles de uso común en Malasia (Tualang, Gelam y Acacia) en relación con sus componentes bioquímicos. Observaron que no existían diferencias significativas en el contenido de fenoles totales entre las mieles, sin embargo, los resultados encontrados presentaron valores más altos que los reportados en mieles provenientes de la India, Argentina y Burkina Faso de África occidental. El contenido de flavonoides totales tampoco presentó diferencias significativas y mostraron valores más altos a los reportados en Portugal y España.

La resistencia a la tracción comúnmente se ha asociado con la organización, el contenido y las propiedades físicas de la red de fibras de colágeno (Dombi et al., 1993). Bajo este contexto, estudios han demostrado que la resistencia a la tracción en heridas también difiere según el tipo de miel utilizada ya que su acción sobre el colágeno en el proceso de cicatrización muestra diferencias. Por otra parte, Rozaini et al. (2004), observaron que la miel de Manuka, Durio Zibethinus (Durian) y Ananas comosus (Nenas) presentaban una mayor resistencia a la tracción en comparación con heridas tratadas con otras dos mieles, Melaleuca (Gelam) y Cocos nucifera (Kelapa), infiriendo que esto es posible gracias a que el primer grupo es capaz de aumentar el contenido de colágeno y acelerar la maduración del mismo. Nisbet et al. (2010), demostraron que el nivel de colágeno y de hidroxiprolina en los grupos tratados con miel monofloral de castaño (Castanea sativa), rododendro (Rhododendron lúteo) y miel multifloral, provenientes de la región del Mar Negro de Turquía, eran significativamente más altos que en el grupo control no tratado. En este estudio, no se detectaron diferencias significativas entre los distintos tipos de miel, pero se observaron menos zonas sin cicatrizar en las heridas del grupo de miel multifloral en los días 7 y 14 post lesión. Por su parte, Schencke et al. (2015) observaron que el tratamiento de heridas por quemaduras con miel monofloral de Ulmo (Eucryphia cordifolia) suplementada con ácido ascóbico, promueve la producción de fibras colágenas y rápido proceso de cicatrización. A través del método Sirius Red (Montes
\& Junqueira, 1991) observaron que las fibras colágenas se distribuían como haces paralelos al tejido epitelial recién formado, logrando una alta densidad. En relación al tipo de colágeno, Velnar et al. indicaron que la dermis no herida contiene un $80 \%$ de colágeno tipo I y $25 \%$ de colágeno tipo III, mientras que en las heridas el tejido de granulación expresa un $40 \%$ de colágeno tipo III. Schencke et al. (2015), demostraron la presencia de colágeno tipo III en toda la zona cicatrizal de la dermis de heridas tratados con miel de Ulmo y suplementada con ácido ascóbico, correlacionándose con lo descrito por Benavides (2008), quién señaló que este colágeno se secreta sólo en estadios tempranos de la reparación, siendo máximo entre los días cinco a siete. El colágeno tipo I también estaba presente en la dermis cicatrizal, tornándose más denso y con una distribución tridimensional hacia la hipodermis.

\section{Desventajas (alergias o efectos nocivos)}

Debido a los beneficios de la miel en el tratamiento de heridas y quemaduras, su uso clínico como un agente tópico continua validándose. Se ha evaluado su toxicidad en queratinocitos y fibroblastos, mostrando que la miel puede ser clasificada como una sustancia no tóxica, y que puede ser utilizada con seguridad no sólo para aplicaciones externas sobre la piel sana, sino también, como un vendaje sobre heridas (Ranzato et al., 2012).

Sin embargo, es importante considerar que la miel podría producir intoxicaciones sistémicas debido a la probable presencia de endosporas latentes de Clostridium botulinum. Por este motivo, la miel de uso terapéutico se esteriliza a través de la radiación gamma, que destruye las esporas bacterianas conservando la actividad biológica y enzimática. Es interesante señalar que las desventajas de su uso tópico son relativamente menores, sin informes de alergias o alteración de la glicemia en pacientes diabéticos. Aunque la alergia a la miel es poco común, se señala que las proteínas del polen en productos cosméticos, a base de miel, podrían funcionar como sensibilizadores en personas alérgicas (Meda et al., 2004; Burlando \& Cornara, 2013).

\section{CONCLUSIONES}

En esta revisión se destacan las investigaciones que evidencian las propiedades terapéuticas de la miel y sus posibles mecanismos de acción, demostrando la eficacia de la miel en el tratamiento de heridas y quemaduras. La miel es un agente terapéutico tópico ideal, ya que no se adhiere a la superficie de la herida. Además de presentar una acción anti-bacteriana natural contra la mayoría de las 
heridas infectadas, tiene actividad anti-oxidante, antiinflamatoria, y de desbridamiento. Al parecer cada uno de sus componentes actúan en conjunto participando de manera sinérgica para lograr la cicatrización total de la herida. Por otra parte, las propiedades físicas de la miel crean una barrera y un medio ambiente local húmedo que permite el desbridamiento eliminando el tejido necrótico y la escara, favoreciendo la cicatrización de heridas. Las propiedades cicatrizantes de la miel le permiten promover la angiogénesis, granulación y epitelización, estimular linfocitos, fagocitos, fibroblastos y queratinocitos y, modular la síntesis de colágeno y su maduración. Su acción la realiza en todas las etapas del proceso fisiológico de reparación logrando acelerar la cicatrización de heridas y mejorar la resistencia a la tracción. Es importante considerar que la elección de la miel para su uso terapéutico en heridas de distintas etiologías dependerá de su origen floral y ubicación geográfica, ya que las mieles producidas tendrán diferentes composiciones y, por tanto, diferente valor medicinal. Finalmente, en acuerdo a lo señalado en la literatura consultada, concluimos que el uso clínico de la miel como agente tópico ofrece una buena alternativa, ya que no produce efectos secundarios significativos, es efectivo y de bajo costo.

SCHENCKE, C.; VÁSQUEZ, B.; SANDOVAL, C. \& DEL SOL, M. The role of honey in the morphophysiologic processes of wound repair. Int. J. Morphol., 34(1):385-395, 2016.

SUMMARY: The benefits of honey bee honey (Apis mellifera) have been known for thousands of years and it has been used since ancient times for its nutritional and medicinal values. It was "rediscovered" by modern medicine as a result of its important antibacterial properties in infected wounds. New studies have argued for the ability of honey to interact with the complex cellular machinery and achieve tissue repair. Therefore, a review was carried out of current contents of the properties of honey and their role in the morphophysiological processes of wound repair. Hence, antibacterial action, anti-oxidant and anti-inflammatory components of its properties were evaluated. At the same time, we reviewed physical and wound debridement benefits, the importance of floral origin and geographical location in the medicinal value of different types of honey. The studies suggest that the effects of honey are the result of a combined activity, where its components interact synergistically to achieve therapeutic function. Its action is realized in all stages of the morphophysiological repair process, accomplishing accelerated wound healing. The floral origin and geographical location of the honey are important factors to consider since the composition of honey is variable, as is its medicinal value.

KEY WORDS: Wounds; Burns; Wound repair; Honey.

\section{REFERENCIAS BIBLIOGRÁFICAS}

Abuharfeil, N.; Al-Oran, R. \& Abo-Shehada, M. The effect of bee honey on the proliferative activity of human Band T-lymphocytes and the activity of phagocytes. Food Agric. Immunol., 11(2):169-77, 2010.

Adams, C. J.; Boult, C. H.; Deadman, B. J.; Farr, J. M.; Grainger, M. N.; Manley-Harris, M. \& Snow, M. J. Isolation by HPLC and characterisation of the bioactive fraction of New Zealand Manuka (Leptospermum scoparium) honey. Carbohydr. Res., 343(4):651-9, 2008.

Ahmed, S. \& Othman, N. H. Review of the medicinal effects of tualang honey and a comparison with manuka honey. Malays. J. Med. Sci., 20(3):6-13, 2013.

Al-Jadi, A. M.; Enchang, F. K. \& Yusoff, K. M. The effect of Malaysian honey and its major components on the proliferation of cultured fibroblasts. Turk. J. Med. Sci., 44(5):733-40, 2014.
Al-Waili, N. S.; Salom, K.; Butler, G. \& Al Ghamdi, A. A. Honey and microbial infections: a review supporting the use of honey for microbial control. J. Med. Food, 14(10):1079-96, 2011.

Aljady, A. M.; Kamaruddin, M. Y.; Jamal, A. M. \& Mohd Yassim, M. Y. Biochemical study on the efficacy of malaysian honey on inflicted wounds: an animal model. Med. J. Islamic Acad. Sci., 13(3):125-32, 2000.

Alzahrani, H. A.; Boukraa, L.; Bellik, Y.; Abdellah, F.; Bakhotmah, B. A.; Kolayli, S. \& Sahin, H. Evaluation of the antioxidant activity of three varieties of honey from different botanical and geographical origins. Glob. J. Health Sci., 4(6):191-6, 2012.

Anklam, E. A review of the analytical methods to determine the geographical and botanical origin of honey. Food Chem., 63(4):549-62, 1998. 
Archambault, D.; Morin, G. \& Elazhary, M. A. Effect of sodium diethyldithiocarbamate, Corynebacterium parvum and mycobacterial cell wall extract in vitro blastogenic responses of bovine blood lymphocytes. Cornell Vet., 79(1):11-24, 1989.

Bean, A.; Molan, P. \& Cursons, R. Anti-inflammatory proteins and methods of preparation and use thereof. World Intellectual Propery Organisation, WO/2012/087160A2, 2012.

Benavides, J. Reparación de heridas cutáneas. Rev. Asoc. Col. Dermatol., 16(1):29-35, 2008.

Blasa, M.; Candiracci, M.; Accorsi, A.; Piacentini, M. P.; Albertini, M. C. \& Piatti, E. Raw Millefiori honey is packed full of antioxidants. Food Chem., 97(2):217-22, 2006.

Boekema, B. K.; Pool, L. \& Ulrich, M. M. The effect of a honey based gel and silver sulphadiazine on bacterial infections of in vitro burn wounds. Burns, 39(4):754-9, 2013.

Brownlee, M. Advanced protein glycosylation in diabetes and aging. Annu. Rev. Med., 46(1):223-34, 1995.

Burlando, B. \& Cornara, L. Honey in dermatology and skin care: a review. J. Cosmet. Dermatol., 12(4):306-13, 2013.

Butchko, G. M.; Armstrong, R. B.; Martin, W. J. \& Ennis, F. A. Influenza A viruses of the $\mathrm{H} 2 \mathrm{~N} 2$ subtype are lymphocyte mitogens. Nature, 271:66-7, 1978.

Calderón, M. D.; Figueroa, C. S.; Arias, J. S.; Sandoval, A. H. \& Torre, F. O. Combined therapy of Ulmo honey (Eucryphia cordifolia) and ascorbic acid to treat venous ulcers. Rev. Latino-Am. Enfermagem, 23(2):259-66, 2015.

Cushnie, T. P. \& Lamb, A. J. Antimicrobial activity of flavonoids. Int. J. Antimicrob. Agents, 26(5):343-56, 2005.

Chua, L. S.; Rahaman, N. L. A.; Adnan, N. A. \& Tan, T. T. E. Antioxidant activity of three honey Samples in relation with their biochemical components. J. Anal. Methods Chem., 2013:313798, 2013.

Dobre, I.; Gâdei, G.; Pâtrascu, L.; Elisei, A. M. \& Segal, R. The antioxidant activity of selected Romanian honeys. AUDJG - Food Technology, 34(2):67-73, 2010.

Dombi, G. W.; Haut, R. C. \& Sullivan, W. G. Correlation of highspeed tensile strength with collagen content in control and lathyritic rat skin. J. Surg. Res., 54(1):21-8, 1993.

Eliopoulos, G. M. \& Eliopoulos, C. T. Antibiotic combinations: should they be tested?. Clin. Microbiol. Rev., 1(2):13956,1988 .
Estrada, H.; Gamboa, M.; Chaves, C. \& Arias, M. Evaluación de la actividad antimicrobiana de la miel de abeja contra Staphylococcus aureus, Staphylococcus epidermidis, Pseudomonas aeruginosa, Escherichia coli, Salmonella enteritidis, Listeria monocytogenes y Aspergillus niger. Evaluación de su carga microbiológica. Arch. Latin. Nutr., 55(2):167-71, 2005.

Eteraf-Oskouei, T. \& Najafi, M. Traditional and modern uses of natural honey in human diseases: A review. Iran J. Basic Med. Sci., 16(6):731-42, 2013.

Frankel, S.; Robinson, G. E. \& Berenbaum, M. R. Antioxidant capacity and correlated characteristics of 14 unifloral honeys. J. Apicult. Res., 37(1):27-31, 1998.

Gheldof, N.; Wang, X. H. \& Engeseth, N. J. Identification and quantification of antioxidant components of honeys from various floral sources. J. Agric. Food Chem., 50(21):5870-7, 2002.

Godebo, G.; Kibru, G. \& Tassew, H. Multidrug-resistant bacterial isolates in infected wounds at Jimma University Specialized Hospital, Ethiopia. Ann. Clin. Microbiol. Antimicrob., 12:17, 2013.

Gupta, S. S.; Singh, O.; Bhagel, P. S.; Moses, S.; Shukla, S. \& Mathur, R. K. Honey dressing versus silver sulfadiazene dressing for wound healing in burn patients: A Retrospective Study. J. Cutan. Aesthet. Surg., 4(3):183-7, 2011.

Hadagali, M. D. \& Chua, L. S. The anti-inflammatory and wound healing properties of honey. Eur. Food Res. Technol., 239(6):1003-14, 2014.

Hussein, S. Z.; Mohd Yusoff, K.; Makpol, S. \& Mohd Yusof, Y. A. Gelam honey inhibits the production of proinflammatory, mediators NO, PGE(2), TNF- $\alpha$, and IL6 in carrageenan-induced acute paw edema in rats. Evid.Based Complement. Alternat. Med., 2012:109636, 2012.

Jenkins, R. E. \& Cooper, R. Synergy between oxacillin and manuka honey sensitizes methicillin-resistant Staphylococcus aureus to oxacillin. J. Antimicrob. Chemother., 67(6):1405-7, 2012.

Jubri, Z.; Rahim, N. B. \& Aan, G. J. Manuka honey protects middle-aged rats from oxidative damage. Clinics (São Paulo), 68(11):1446-54, 2013.

Jull, A. B.; Cullum, N.; Dumville, J. C.; Westby, M. J.; Deshpande, S. \& Walker, N. Honey as a topical treatment for wounds. Cochrane Database Syst. Rev., 3:CD005083, 2015.

Kandil, A.; Elbanby, M.; Abd-Elwahed, K.; Sehly, G. A. \& Ezzet, N. Healing effect of true floral and false nonfloral honey on medical wounds. J. Drug. Res. Egypt, 17(1-2):716, 1987. 
Khoo, Y. T.; Halim, A. S.; Singh, K. K. \& Mohamad, N. A. Wound contraction effects and antibacterial properties of Tualang honey on full-thickness burn wounds in rats in comparison to hydrofibre. BMC Complement. Altern. Med., 10:48, 2010.

Krpan, M.; Markovic, K.; Saric, G.; Skoko, B.; Hruskar, M. \& Vahcic, N. Antioxidant activities and total phenolics of acacia honey. Czech J. Food Sci., 27:S245-7, 2009.

Kwakman, P. H.; te Velde, A. A.; de Boer, L.; Speijer, D.; Vandenbroucke-Grauls, C. M. \& Zaat, S. A. How honey kills bacteria. FASEB J., 24(7):2576-82, 2010.

Lay-flurrie, K. Honey in wound care: effects, clinical application and patient benefit. Br. J. Nurs., 17(11):S30, 2008.

Lu, J.; Carter, D. A.; Turnbull, L.; Rosendale, D.; Hedderley, D.; Stephens, J.; Gannabathula, S.; Steinhorn, G.; Schlothauer, R. C.; Whitchurch, C. B. \& Harry, E. J. The effect of New Zealand kanuka, manuka and clover honeys on bacterial growth dynamics and cellular morphology varies according to the species. PLOS ONE, 8(2):e55898, 2013.

Lu, J.; Turnbull, L.; Burke, C. M.; Liu, M.; Carter, D. A.; Schlothauer, R. C.; Whitchurch, C. B. \& Harry, E. J. Manuka-type honeys can eradicate biofilms produced by Staphylococcus aureus strains with different biofilmforming abilities. PeerJ, 2:e326, 2014.

Lusby, P. E.; Coombes, A. \& Wilkinson, J. M. Honey: a potent agent for wound healing?. J. Wound Ostomy Continence Nurs., 29(6):295-300, 2002.

Majtán, J.; Kovácová, E.; Bíliková, K. \& Simúth, J. The immunostimulatory effect of the recombinant apalbumin 1-major honeybee royal jelly protein-on TNFalpha release. Int. Immunopharmacol., 6(2):269-78, 2006.

Majtán, J.; Kumar, P.; Majtán, T.; Walls, A. F. \& Klaudiny, J. Effect of honey and its major royal jelly protein 1 on cytokine and MMP-9 mRNA transcripts in human keratinocytes. Exp. Dermatol., 19(8):e73-9, 2010.

Meda, A.; Lamien, C. E.; Millogo, J.; Romito, M. \& Nacoulma, O. G. Therapeutic uses of honey and honeybee larvae in central Burkina Faso. J. Ethnopharmacol., 95(1):103-7, 2004.

Meda, A.; Lamien, C. E.; Romito, M.; Millogo, J. \& Nacoulma, O. G. Determination of the total phenolic, flavonoid and proline contents in Burkina Fasan honey, as well as their radical scavenging activity. Food Chem., 91(3):571-7, 2005.
Melake, N. A.; Eissa, N. A.; Keshk, T. F. \& Sleem, A. S. Prevalence of multidrug-resistant bacteria isolated from patients with burn infection. Menoufia Med. J., 28(3):67784, 2015.

Molan, P. Why honey is effective as a medicine. 2. The scientific explanation of its effects. Bee World, 82(1):2240, 2001.

Molan, P. Re-introducing honey in the management of wounds and ulcers - theory and practice. Ostomy Wound Manage, 48(11):28-40, 2002.

Molan, P. Debridement of wounds with honey. J. Wound Technol., 5:12-7, 2009.

Molan, P. The evidence and the rationale for the use of honey as a wound dressing. Wound Practice \& Research, 19(4):204-20, 2011.

Molan, P. \& Rhodes, T. Honey: A biologic wound dressing. Wounds, 27(6):141-51, 2015.

Montenegro, G. \& Ortega, X. Usos del extracto de miel unifloral de ulmo como bactericida y fungicida. World Intellectual Propery Organisation, WO/2011/057421, 2011.

Montes, G. S. \& Junqueira, L. C. The use of the Picrosiriuspolarization method for the study of the biopathology of collagen. Mem. Inst. Oswaldo Cruz, 86(Suppl3):1-11, 1991.

Mujalde, V. S.; Jalaj, A. \& Raj pipariya, P. To Assess the efficacy of honey in comparison with $1 \%$ silver sulfadiazine cream as a burn wound dressing in superficial and partial thickness of burns. Sch. J. App. Med. Sci., 2(1B):193-6, 2014.

Müller, P.; Alber, D. G.; Turnbull, L.; Schlothauer, R. C.; Carter, D. A.; Whitchurch, C. B \& Harry, E. J. Synergism between Medihoney and Rifampicin against Methicillin-Resistant Staphylococcus aureus (MRSA). PLoS ONE, 8(2):e57679, 2013.

Nafea, E. A.; Moselhy, W. A. \& Fawzy, A. Does the HMF value affect the antibacterial activity of the bee honey. Egypt Acad. J. Biol. Sci., 4:13-9, 2011.

Nakajima, Y.; Nakano, Y.; Fuwano, S.; Hayashi, N.; Hiratoko, Y.; Kinoshita, A.; Miyahara, M.; Mochizuki, T.; Nishino, K.; Tsuruhara, Y.; Yokokawa, Y.; Iuchi, T.; Kon, Y.; Mukai, K.; Kitayama, Y.; Murakado, N.; Okuwa, M. \& Nakatani, T. Effects of three types of japanese honey on full-thickness wound in mice. Evid.-Based Complement. Alternat. Med., 2013:504537, 2013. 
Nisbet, H. O.; Nisbet, C.; Yarim, M.; Guler, A. \& Ozak, A. Effects of three types of honey on cutaneous wound healing. Wounds, 22(11):275:83, 2010.

Nishio, E. K.; Ribeiro, J. M.; Oliveira, A. G.; Andrade, C. G. T. J.; Proni, E. A.; Kobayashi, R. K. T. \& Nakazato, G. Antibacterial synergic effect of honey from two stingless bees: Scaptotrigona bipunctata Lepeletier, 1836, and $S$. postica Latreille, 1807. Sci. Rep., 6:21641, 2016.

Oryan, A.; Alemzadeh, E. \& Moshiri, A. Biological properties and therapeutic activities of honey in wound healing: A narrative review and meta-analysis. J. Tissue Viability, 2016.

Oudrhiri, N.; Farcet, J. P.; Gourdin, M. F.; Divine, M.; Marolleau, J. P.; Bouguet, J.; Lecouedic, J. P.; Shaw, A.; Fradelizi, D. \& Reyes, F. Activation by PHA of CD8 lymphocytes into clonal colony forming cells: Role of interleukin-1. J. Immunol. Methods, 110(2):241-9, 1988.

Pontis, J. A.; Costa, L. A. M. A. da; Silva, S. J. R. da \& Flach, A. Color, phenolic and flavonoid content, and antioxidant activity of honey from Roraima, Brazil. Food Sci. Technol. (Campinas), 34(1):69-73, 2014.

Pourreza, N. Phenolic Compounds as potential antioxidant. Jundishapur J. Nat. Pharm. Prod., 8(4):149-50, 2013.

Ranzato, E.; Martinotti, S. \& Burlando, B. Epithelial mesenchymal transition traits in honey-driven keratinocyte wound healing: Comparison among different honeys. Wound Repair Regen., 20(5):778-85, 2012.

Raynaud, A.; Ghezali, L.; Gloaguen, V.; Liagre, B.; Quero, F. \& Petit, J. M. Honey-induced macrophage stimulation: AP- 1 and NF- $k$ B activation and cytokine production are unrelated to LPS content of honey. Int. Immunopharmacol., 17(3):874-9, 2013.

Rodriguez-Otero, J. L.; Paseiro, P.; Simal, J. \& Cepeda, A. Mineral-content of the honeys produced in Galicia (NorthWest Spain). Food Chem., 49(2):169-71, 1994.

Rossiter, K.; Cooper, A. J.; Voegeli, D. \& Lwaleed, B. A. Honey promotes angiogeneic activity in the rat aortic ring assay. J. Wound Care, 19(10):440, 2010.

Rozaini, M.Z., Zuki, A.B.Z., Noordin, M., Norimah, Y., Hakim, A.N. The effects of different types of honey on tensile strength evaluation of burn wound tissue healing. Int. J. App. Res. Vet. Med., 2:290-6, 2004.

Saric, G.; Markovic, K.; Vukicevic, D.; Lez, E.; Hruskar, M. \& Vahcic N. Changes of antioxidant activity in honey after heat treatment. Czech J. Food Sci., 31(6):601-6, 2013.
Schencke, C.; Salvo, J.; Vasconcellos, A. \& del Sol, M. Estudio comparativo de la cicatrización en quemaduras con tratamiento en base a miel de ulmo (Eucryphia cordifolia) y vitamina $\mathrm{C}$ oral versus hidrogel en cobayos (Cavia porcellus). Int. J. Morphol., 31(3):839-44, 2013.

Schencke, C.; Vasconcellos, A.; Salvo, J.; Veuthey, C. \& del Sol, M. Efecto cicatrizante de la miel de ulmo (Eucryphia cordifolia) suplementada con ácido ascórbico como tratamiento en quemaduras. Int. J. Morphol., 33(1):137-43, 2015.

Sherlock, O.; Dolan, A.; Athman, R.; Power, A.; Gethin, G.; Cowman, S. \& Humphreys, H. Comparison of the antimicrobial activity of Ulmo honey from Chile and Manuka honey against methicillin-resistant Staphylococcus aureus, Escherichia coli and Pseudomonas aeruginosa. BMC Complement. Alternat. Med., 1O(1):47, 2010.

Singh, M. P.; Chourasia, H. R.; Agarwal, M.; Malhotra, A.; Sharma, M.; Sharma, D. \& Khan, S. Honey as complementary medicine: A review. IJPBS, 3(2):12-31, 2012.

Subrahmanyam, M. Topical application of honey for burn wound treatment - an overview. Ann. Burns Fire Disasters, 20(3):137-9, 2007.

Subrahmanyam, M.; Shahapure, A. G.; Nagne, N. S.; Bhagwat, V. R. \& Ganu, J. V. Effects of topical application of honey on burn wound healing. Ann. Burns Fire Disasters, 14:143-5, 2001.

Suguna, L.; Chandrakasan, G.; Joseph, K. T. Influence of honey on collagen metabolism during wound healing in rats. J. Clin. Biochem. Nutr., 13(1):7-12, 1992.

Suguna, L.; Chandrakasan, G.; Ramamoorthy, U. \& Joseph, $\mathrm{K}$. T. Influence of honey on biochemical and biophysical parameters of wounds in rats. J. Clin. Biochem. Nutr., 14(2):91-9, 1993.

Talebi, A.; Torgerson, P. R. \& Mulcahy, G. Optimal conditions for measurement of blastogenic responses of chickens to Concanavalin-A in whole-blood assays. Vet. Immunol. Immunop., 46(3-4):293-301, 1995.

Tan, M. K.; Hasan Adli, D. S.; Tumiran, M. A.; Abdulla, M. A. \& Yusoff, K. M. The efficacy of gelam honey dressing towards excisional wound healing. Evid.-Based Complement. Alternat. Med., 2012:805932, 2012.

Tonks, A.; Cooper, R. A.; Price, A. J.; Molan, P. C. \& Jones, K. P. Stimulation of TNF-alpha release in monocytes by honey. Cytokine, 14(4):240-2, 2001. 
SCHENCKE, C.; VÁSQUEZ, B.; SANDOVAL, C. \& DEL SOL, M. El rol de la miel en los procesos morfofisiológicos de reparación de heridas. Int. J. Morphol., 34(1):385-395, 2016.

Tonks, A. J.; Dudley, E.; Porter, N. G.; Parton, J.; Brazier, J.; Smith, E. L. \& Tonks, A. A 5.8-kDa component of manuka honey stimulates immune cells via TLR4. J. Leukoc. Biol., 82(5):1147-55, 2007.

Ulrich-Merzenich, G.; Panek, D.; Zeitler, H.; Vetter, H. \& Wagner, H. Drug development from natural products: exploiting synergistic effects. Indian J. Exp. Biol., 48(3):208-19, 2010.

Vandamme, L.; Heyneman, A.; Hoeksema, H.; Verbelen, J. \& Monstrey, S. Honey in modern wound care: a systematic review. Burns, 39(8):1514-25, 2013.

Velnar, T.; Bailey, T. \& Smrkolj, V. The wound healing process: an overview of the cellular and molecular mechanisms. $J$. Int. Med. Res., 37(5):1528-42, 2009.

Venema, D. P.; Hollman, P. C. H.; Janssen, K. P. L. T. M. \& Katan, M. B. Determination of acetylsalicylic acid and salicylic acid in foods, using HPLC with fluorescence detection. J. Agric. Food Chem., 44(7):1762-7, 1996.

Winter, G. D. Formation of the scab and the rate of epithelialization of the young domestic pig. Nature, 193:293-4, 1962.

Yaghoobi, R.; Kazerouni, A. \& Kazerouni, O. Evidence for clinical use of honey in wound healing as an anti-bacterial, antiinflammatory anti-oxidant and anti-viral agent: a review. Jundishapur J. Nat. Pharm. Prod., 8(3):100-4, 2013.

Zbuchea, A. Up-to-date use of honey for burns treatment. Ann. Burns Fire Disasters, 27(1):22-30, 2014.

\section{Dirección para correspondencia: \\ Mg. Cs. Carolina Schencke \\ Programa de Doctorado \\ Ciencias Morfológicas \\ Facultad de Medicina \\ Universidad de La Frontera \\ Temuco - CHILE}

\section{Email: c.schencke01@ufromail.cl carolinaschencke@gmail.com}

Recibido : 22-12-2015
Aceptado:18-01-2016 